The use and characterization of a backilluminated charge-coupled device in investigations of pulsed $x$-ray and radiation sources

Wilfred Fullagar, Jens Uhlig, Monika Walczak, Sophie Canton, and Villy Sundström

Citation: Review of Scientific Instruments 79, 103302 (2008); doi: 10.1063/1.3000003

View online: http://dx.doi.org/10.1063/1.3000003

View Table of Contents: http://aip.scitation.org/toc/rsi/79/10

Published by the American Institute of Physics

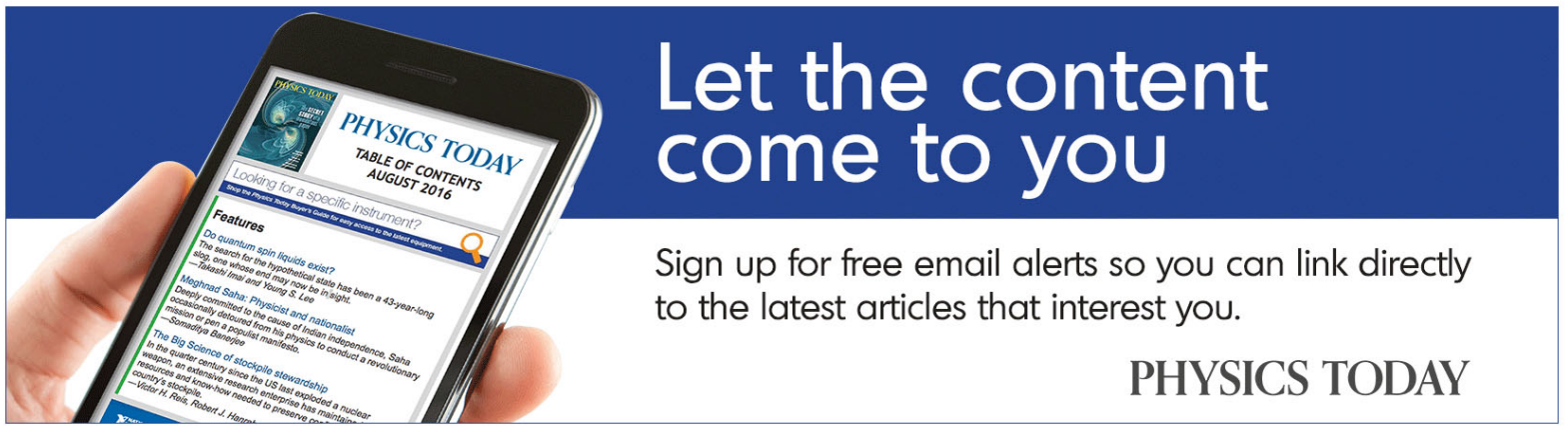




\title{
The use and characterization of a backilluminated charge-coupled device in investigations of pulsed $\mathrm{x}$-ray and radiation sources
}

\author{
Wilfred Fullagar, ${ }^{1,2}$ Jens Uhlig, ${ }^{1}$ Monika Walczak, ${ }^{1}$ Sophie Canton, ${ }^{1}$ and Villy Sundström ${ }^{1}$ \\ ${ }_{1}^{1}$ Division of Chemical Physics, Kemicentrum Lund University, P.O. Box 124, SE-22100 Lund, Sweden \\ ${ }^{2}$ Monash Centre for Synchrotron Science, Monash University, Clayton, VIC 3800, Australia
}

(Received 7 April 2008; accepted 23 September 2008; published online 29 October 2008)

\begin{abstract}
Examinations of bremsstrahlung and energetic electron beams from a novel laser plasma source motivate and assist characterization of a backthinned, backilluminated direct detection x-ray charge-coupled device (CCD), a topology that is uncommon in hard x-ray work. Behavior toward pseudomonochromatic $\left({ }^{55} \mathrm{Fe}\right)$ and multichromatic $\left({ }^{241} \mathrm{Am}\right)$ sources is briefly reviewed under optimized noise conditions. Results collectively establish the previously unknown functional depth structure. Several modes of usage are illustrated in $\sim 4-20 \mathrm{keV} x$-ray laser plasma source investigations, where the significance of the characterization is briefly discussed. The spectral redistribution associated with this CCD topology is unfavorable, yet appropriate analysis ensures that sufficient spectral information remains for quantitative determination of broadband $\mathrm{x}$-ray flux and spectra in essentially single laser shot measurements. The energy dependence of nascent electron cloud radii in silicon is determined using broadband x-rays from the laser plasma source, turning the narrow depletion depth to advantage. Finally, the characterization is used to quantify recent X-ray spectral explorations of the water jet laser plasma source operating under aspirator vacuum. These results will have key value for establishment of laboratory based ultrafast extended $\mathrm{x}$-ray absorption fine structure experiments using microbolometric detectors. (C) 2008 American Institute of Physics. [DOI: 10.1063/1.3000003]
\end{abstract}

\section{INTRODUCTION}

Charge-coupled devices (CCDs) are a mature technology. ${ }^{1}$ The use of direct detection CCDs is fairly widespread in $\mathrm{x}$-ray communities requiring single photon sensitivity at energies up to $\sim 20 \mathrm{keV}$ and moderate resolution of photon energies within each pixel. They permit high spatial resolution imaging capability with the convenience of electronic data acquisition, making them a popular choice for x-ray studies in plasma physics, ${ }^{2}$ astronomy, ${ }^{3,4} \mathrm{x}$-ray imaging, ${ }^{5,6}$ and diffraction ${ }^{7}$ applications. Many accounts of direct detection CCDs in a laser plasma context have substantially attended to observation of x-ray emission lines, ${ }^{2,8,9}$ or their use in spectral imaging modes. ${ }^{10,11}$ Often, an understanding of the $\mathrm{x}$-ray source is sought, with limited attention to detector details.

A backilluminated, non-deep-depleted direct detection $\mathrm{CCD}$ has been available to us for the evaluation of radiations from a novel laser plasma source. ${ }^{12}$ Its extended use ${ }^{13-16}$ in a fundamental research environment ${ }^{17}$ has led to a continual demand for its characterization, in the face of limited technical information about the particular device. Its topology is unusual in the hard $\mathrm{x}$-ray field of study. We evaluate its energy resolution limit, strong $\mathrm{x}$-ray spectral redistribution and declining sensitivity to high energy $\mathrm{x}$-rays using pseudomonochromatic and multichromatic sources. These allow an outline of its depth structure that are subsequently fine-tuned using results from our broadband laser plasma $\mathrm{x}$-ray source, with calibration by a Ge point detector. The same measurements allow estimation of thermalized electron cloud radii over a continuum of $\mathrm{x}$-ray energies, with a simple and empirical outcome that may find applicability from visible energies to $\sim 60 \mathrm{keV}$. Several important plasma source experiments that have benefited from the CCD characterization are illustrated. Finally, we use our findings to quantitatively measure $\sim 4-20 \mathrm{keV}$ ultrafast broadband $\mathrm{x}$-ray spectra that should be obtainable using kilohertz repetition laser systems in developments that are currently underway. Detector limitations and their causes realized in this work led us to suggest the incorporation of microbolometer arrays in associated ultrafast molecular structure dynamics developments. ${ }^{12}$

An overview of the processes occurring during direct $\mathrm{x}$-ray detection indicates the limitations encountered in this work. The photoelectric cross section dominates the interaction of $\mathrm{x}$ rays with $\mathrm{Si}$ (the $\mathrm{CCD}$ material) for $\mathrm{x}$-ray energies up to $\sim 60 \mathrm{keV} .{ }^{18}$ The initial outcome is an ionized atom and an energetic photoelectron. The former may generate further electrons by Auger and shakeup processes, while these new electrons and the energetic photoelectron cause further excitations on length scales of approximately a micron and less. ${ }^{19}$ The electronically measurable outcome is a localized cloud of thermalized electrons in the semiconductor's conduction band. Thermalized electron cloud radii are an increasing function of x-ray photon energy. The clouds diffuse in all directions, following local electric field gradients. Another relaxation channel for the excited atom is x-ray fluorescence; energy that escapes detection by this mechanism gives rise to escape peaks. Also, if x-ray photon capture occurs sufficiently close to the surface, nonthermalized electrons can 
carry energy out of the detector as photoelectrons if their energy is still above the material's work function. Recombination or trapping may occur, also it may not be possible to quantitatively bring the entire charge cloud into a readout amplifier. In the course of the overall process, most of the original x-ray energy is in fact converted to heat. The microscopically stochastic nature of the heat versus conduction electron outcome leads to a fundamental (Fano ${ }^{20}$ limit for the energy resolution of nonbolometric detectors. ${ }^{21}$

With these provisos, the number of measurable electrons arising from an event is generally proportional to the original $\mathrm{x}$-ray photon energy so that from a CCD user perspective, and if all goes well, a discrete $\mathrm{x}$-ray event translates to a single bright pixel in the associated CCD readout. The corresponding charge is proportional to the energy of the $\mathrm{x}$-ray photon. A histogram of pixel intensities can be interpreted as the energy spectrum, given allowances for the many potential losses just described, as well as the possibility of pileup. Circumstances permit good signal to noise ratio for these measurements, indeed sufficient to directly appreciate the Fano limit. The CCD pixels, and the event charges they contain, are read out sequentially. This approach is extremely valuable when events strike the detector in different pixels, but simultaneously from an electronic perspective; it enables one-shot spectral assessment of arbitrarily short pulse, noncollimated x-ray sources.

We take a symptomatic approach to the CCD characterization. The reason is simply that the device we possess continues to be extremely versatile in numerous experiments, yet technical information has been scarce. As mentioned at the outset, CCDs are a mature technology so sophisticated and successful models exist for electron cloud diffusion in different detector regions and electronic biasing conditions in various CCD topologies. For these the reader is directed elsewhere. ${ }^{1,22-24}$ Here a general awareness suffices, coupled to practical observation; the outcome is consistent with general expectations. It has long been apparent that the nondeep-depleted, backilluminated topology is not an ideal CCD topology for hard $\mathrm{x}$-ray detection, for reasons that are clear in this work. Consequently, this work presents a rather unique look at the behavior of this CCD topology in the hard $\mathrm{x}$-ray spectral region. We turn its thin depletion layer to advantage when studying the cloud radius versus $\mathrm{x}$-ray photon energy variation. We go on to quantify extremely useful and contemporary laser plasma $\mathrm{x}$-ray results that used the CCD, required its characterization, and could not be generated using other detectors available at the time.

\section{APPARATUS}

The CCD is a backthinned, backilluminated 512 $\times 512$ pixels, $24.8 \mu \mathrm{m} \times 24.8 \mu \mathrm{m}$ direct detection device (model SXTE/CCD-512TKB1, Scientific Imaging Technologies). It lies $10 \mathrm{~mm}$ behind a $250 \mu \mathrm{m}$ Be window in an evacuated, triple Peltier stage, water cooled camera head, and is coupled to a computer controller interface (ST-130, 16 bit, 200000 samples/s, Princeton Instruments). This work uses a stable gain setting corresponding to $\sim 20.2 \mathrm{eV}$ of $\mathrm{X}$-ray energy per analog to digital converter (ADC) unit, cali- brated using emission lines from an ${ }^{55} \mathrm{Fe}$ source. The preamplifier output and ADC input are relatively biased such that null output from the preamplifier is $\sim 120$ ADC units. This provides adequate resolution and dynamic range to characterize noise functions as well as individual x-ray photons, including high energy or pileup events. Specific information regarding $\mathrm{CCD}$ construction and performance has been obscured by the commercial environment surrounding its manufacture and subsequent integration (the CCD manufacturer has ceased operations). This work builds on the following indications. Quantum efficiency curves for an apparently similar CCD (PIXIS-XO 512B) in the $1-9 \mathrm{keV}$ range can be reverse engineered to suggest an attenuating layer of $0.1 \mu \mathrm{m} \mathrm{Si}$ followed by a sensitive depth of $\sim 14.9 \mu \mathrm{m} \mathrm{Si}$. Backilluminated, backthinned CCDs of $\sim 15 \mu \mathrm{m}$ thickness were available from the CCD manufacturer prior to the delivery of our apparatus in 1994. In these, a bias-dependent potential valley extends several microns in the vicinity of the gate electrodes.

A Ge point detector (ORTEC Canberra GUL0105 with EG\&G ORTEC $4293 \mathrm{MCA}$ card) was used to calibrate each of the x-ray sources examined. It is characterized elsewhere. ${ }^{25}$ For present purposes we take its detection efficiency to be $100 \%$ in the entire spectral region examined in this work $(25 \mu \mathrm{m} \mathrm{Be}$ in front of a Ge detector crystal with active thickness of $5 \mathrm{~mm}$ ). Relative to the CCD, it is associated with very little spectral redistribution, but has a relatively poor energy resolution that is a function of shaping times.

The noise floor of the CCD was established, and the detector always operated in this region. In particular, for our examinations of pulsed sources, CCD exposure times can be arbitrarily short. The presented spectral measurements arise from two readout frames of equal exposure; the first (blank) functioning as a flat field correction. A new blank background measurement is made at the commencement of any measurement. Dark current variation is, in fact, low enough that a potentially $\sqrt{2}$ increase in base line width incurred by two measurements does not necessarily justify the flat field subtraction. However, the procedure ensures symmetry of the baseline peak about zero. It also effectively corrects a stickjump problem ${ }^{26}$ with the ADC, since the flaw is uncorrelated with pixel position and falls well within the noise envelope observed in subsequent pixel intensity histograms. The nature of this problem is that as the input voltage to the ADC is continuously increased, the digital output sticks at particular values for longer than it should, eventually jumping over the correct value to the next higher value. This can happen to the extent that certain values of the digital output are observed twice as often as they should be, while adjacent values are not observed at all.

\section{RADIOACTIVE SOURCE WORK}

Figure 1 condenses a representative fraction of the radioactive source work. It shows:

(a) Spectra from ${ }^{55} \mathrm{Fe}$, showing a histogram of all pixel values, another histogram of the same data after thresholding and grouping pixels into events (to reinte- 


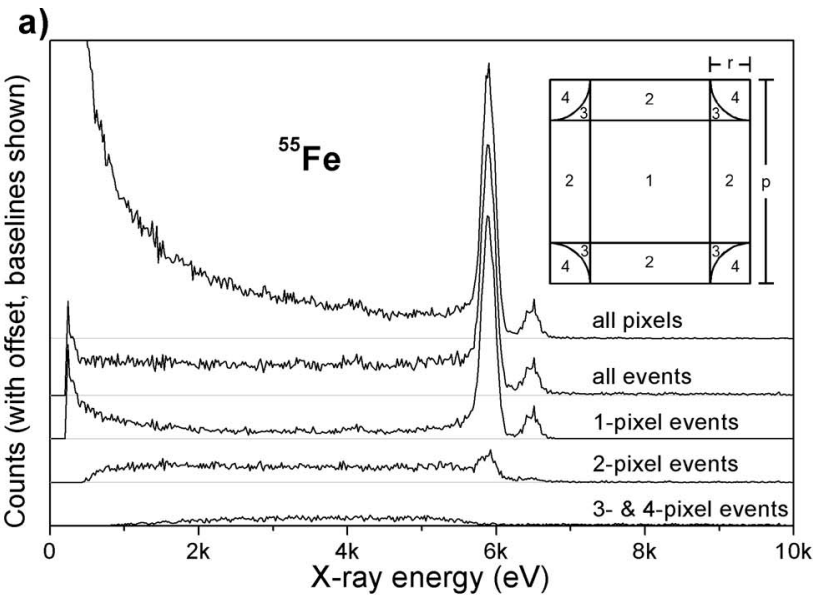

b)

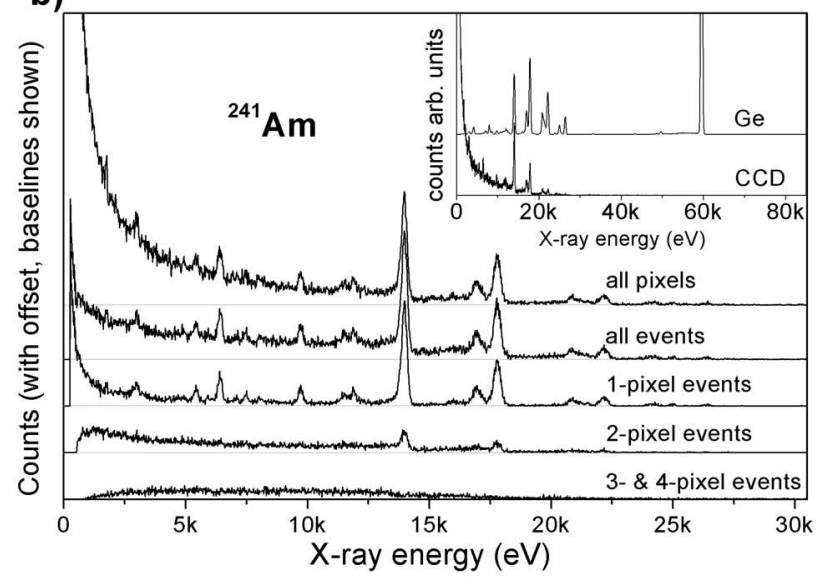

c)
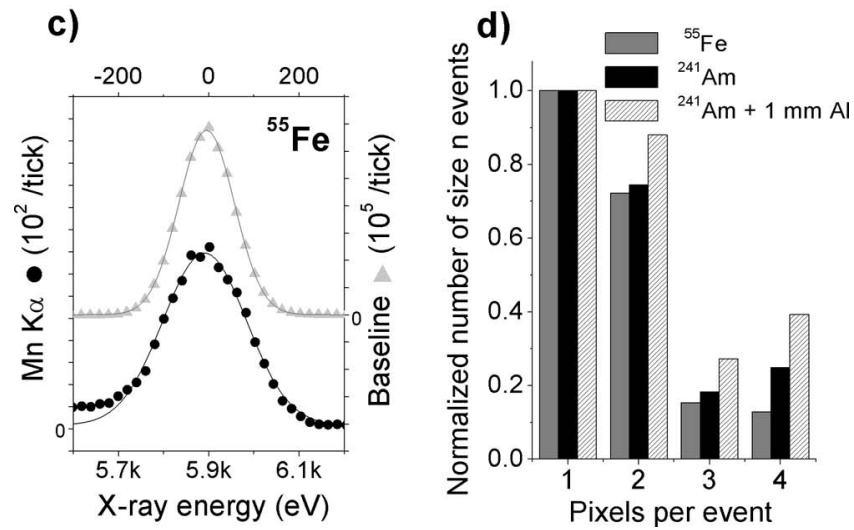

FIG. 1. (a) Pixel histogram, event histogram, and n-pixel event histograms of an ${ }^{55} \mathrm{Fe}$ source measurement. The base line is shown in each case. (b) Analogous histograms from a measurement of an ${ }^{241} \mathrm{Am}$ source. The inset compares the CCD histogram with data obtained using a Ge point detector, showing the rapidly deteriorating high energy response of the CCD. (c) Comparison of the base line and $5.9 \mathrm{keV}$ widths in the same ${ }^{55} \mathrm{Fe}$ data set. (d) High energy events tend to span more pixels. The inset to panel (a) is an aerial sketch of a pixel, showing regions where an event of radius $r$ will span $1,2,3$, and 4 pixels, applicable in the case of fully registered ${ }^{55} \mathrm{Fe}$ events.

grate distributed charge), the histogram of 1 pixel events (those in which thresholded events are in isolated pixels), the histogram of 2 pixel events, and the histogram of 3 and 4 pixel events. A base line is shown in each case. It is clear that 1 pixel events provide the "best" spectrum and that no spectral improvements can be made by consideration of other events.
TABLE I. Event counts obtained by thresholding ${ }^{55} \mathrm{Fe}$ data obtained using the CCD.

\begin{tabular}{lrrccr}
\hline \hline & $\begin{array}{l}\text { 1 pixel } \\
\text { events }\end{array}$ & $\begin{array}{r}\text { 2 pixel } \\
\text { events }\end{array}$ & $\begin{array}{c}\text { 3 and 4 pixel } \\
\text { events }\end{array}$ & $\begin{array}{c}\geq 5 \text { pixel } \\
\text { events }\end{array}$ & Total \\
\hline$\geq 268 \mathrm{eV}$ & 21354 & 15123 & 5514 & 608 & 42599 \\
$\geq 5704 \mathrm{eV}$ & 9343 & 1953 & 1041 & 407 & 12744 \\
$\geq 6856 \mathrm{eV}$ & 28 & 319 & 796 & 336 & 1479 \\
\hline \hline
\end{tabular}

(b) A corresponding set of histograms for ${ }^{241} \mathrm{Am}$. The same deduction can be drawn. Fluorescence and escape peaks are visible as well as the source emission lines. The inset compares the CCD with the Ge detector, showing the former's deteriorating response at higher $\mathrm{x}$-ray energies. A faint trail leading up to but not beyond the $59.5 \mathrm{keV}$ line is visible in the CCD data on close analysis.

(c) A comparison of the spread of empty pixels (base line) with the spread of ${ }^{55} \mathrm{Fe}$ 's $\mathrm{Mn} K_{\alpha}$ line. It is an unresolved doublet (5889.1 and $5900.3 \mathrm{eV}$ ), yet the observed broadening relative to the baseline [full width half maximum (FWHM) of $144 \mathrm{eV}$ ] requires convolution with a Gaussian of FWHM of $161 \mathrm{eV}$ to account for the observed Mn $K \alpha$ breadth of $216 \mathrm{eV}$. This observation guarantees that our measurements are essentially Fano limited. As a result, there is no reason to further pursue questions of electronic noise.

(d) The lateral extent of events increases with the average event energy. All observed events are considered, since it is generally not possible to ascribe the true energy to an arbitrary event in the multiline ${ }^{241} \mathrm{Am}$ spectra. The average event energy from the ${ }^{241} \mathrm{Am}$ source is further raised by filtering; $1 \mathrm{~mm}$ of $\mathrm{Al}$ strongly suppresses the emissions below $\sim 20 \mathrm{keV}$.

(e) (Inset to the ${ }^{55} \mathrm{Fe}$ histograms.) An overhead sketch of a pixel, showing the regions in which an event (assumed circular) will be registered in one, two, 3 or 4 pixels. Because it is not possible to confidently assign the true energy to an arbitrary event in nonmonochromatic spectra, this will only have meaning when applied to the pseudomonochromatic ${ }^{55} \mathrm{Fe}$ data, and events whose total charge corresponds to the known x-ray energy. By classifying events as 1 pixel, 2 pixel, etc., generating a histogram in each case, and counting the number of events that exceed particular thresholds, the numbers of fully registered $n$-pixel events can be established $(>268 \mathrm{eV}=$ above detection noise, between 5704 and $6856 \mathrm{eV}=$ fully registered). Table I shows counts corresponding to these thresholds. The ratios of 1 pixel to 2 pixel to 3 and 4 pixel fully registered events best fits the algebraic ratios derivable from the sketch for a lateral cloud radius of $\sim 1.1 \mu \mathrm{m}$.

The ${ }^{55} \mathrm{Fe}$ histograms in Fig. 1 demonstrate that the partial registry (spectral redistribution to apparently low energies) in our backilluminated CCD has at least two causes. One is the potential for events to span multiple pixels. However, the weakness of the emission line peak in the 2 pixel and 3 and 4 pixel event histograms show that by itself, this 


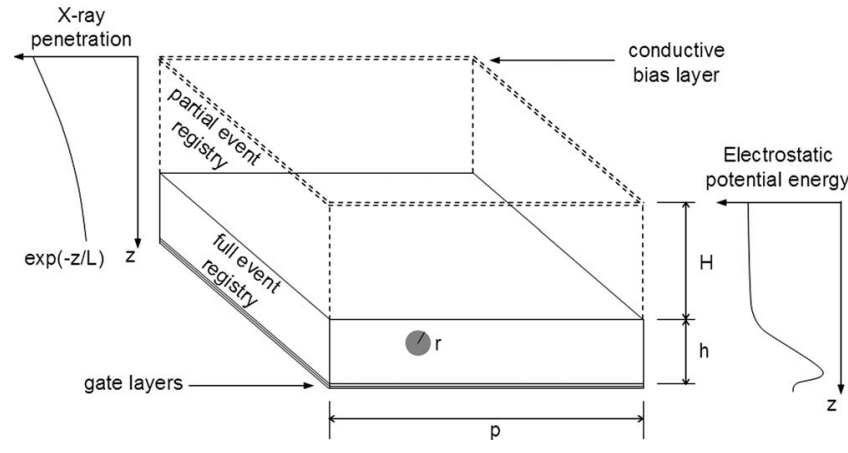

FIG. 2. A simplified sketch of a pixel volume in the CCD. Photons approach from above and are attenuated exponentially. The surface layer $(h)$ is associated with weak field gradients, allowing diffusion of electron clouds both vertically and laterally, and resulting in partial registry of electron clouds forming in this layer. The depletion layer $(h)$ is associated with strong field gradients and a potential minimum (buried channel) in the vicinity of the gate layer. Thermalized electron clouds contained within this layer cannot diffuse out, and are fully registered.

does very little to account for the many partial events. Instead, we see that the more pixels spanned by an event, the lower the likelihood of complete registry at the known peak energy. The same situation clearly also applies to the higher energy ${ }^{241} \mathrm{Am}$ spectra. An explanation ${ }^{27}$ can be found in the presumed depth structure of the CCD, as follows. Seen by the source, and sketched in Fig. 2, our backilluminated topology starts with an $\sim 0.1 \mu \mathrm{m}$ superficial conductive layer that applies the CCD substrate bias. Next comes a layer several microns deep, characterized by weak vertical field gradients, which we refer to as the surface layer. Electron cloud diffusion occurs in all directions in this layer, owing to the weak field gradients. The part of the cloud that diffuses toward the superficial layer is lost as far as measurement is concerned, while lateral diffusion means that the remainder of the cloud can be spread over several pixels. The partial loss of electron clouds in this layer means that the inferred event energies will be falsely low; they have been spectrally redistributed. ${ }^{28}$ The exponentially decaying likelihood of $\mathrm{x}$-ray penetration, and the surface layer's relative thickness leads to numerous partial events and the extensive spectral redistribution observed in our CCD. The problem can be quantified in conjunction with physical models; however, that has not been the objective in the present work. Deeper in the structure (close to the readout gates in the backilluminated device) is the depletion layer. Here the vertical field gradients are sufficient to counter vertical diffusion. Electron clouds formed in this region are quantitatively swept into a potential minimum (buried conduction channel) associated with the gate layer, from whence they are eventually read out with very low losses. ${ }^{1}$

In terms of practical use, it is clear that we must confine our attention to 1 pixel events to best avoid spectral redistribution. We must also accept the reality that some 1 pixel events are also partially registered, to an extent dependent on cloud radius and therefore photon energy. The ${ }^{55} \mathrm{Fe}$ and ${ }^{241}$ Am 1 pixel spectra in Fig. 1 demonstrate these points, showing tolerably low levels of partial registry except at energies in the range $\sim 260-2000 \mathrm{eV}$. Although it is above the readout noise, this region is powerfully attenuated by the
$250 \mu \mathrm{m}$ Be window on the CCD, so there is little risk of confusing these partial events with real events.

Comparison of the total number of ${ }^{55} \mathrm{Fe}$ events observed using the CCD versus the Ge detector (absolute source calibration) confirms the $\sim 15 \mu \mathrm{m}$ total thickness of the $\mathrm{Si}$ in our CCD. The number of fully versus partially registered events (Table I) then gives a first estimate of the surface and depletion layer thicknesses. They are $\sim 10.6$ and $\sim 4.4 \mu \mathrm{m}$, respectively, here with the assumption of negligible charge cloud radii.

Observable cloud size is a function of where in the CCD's depth the cloud forms and the nature of the measurement. Depth and lateral field gradients are functions of three dimensional location in the CCD structure, as is readily appreciated from Fig. 2. Depth field gradients tend to be strong compared to lateral gradients, so given otherwise isotropic diffusion, electron cloud sizes inferred from depth measurements are generally smaller. As we have seen, lateral measurements are based on the charge cloud spanning multiple pixels. On the other hand, depth measurements must rely on how much of the charge cloud falls within the depletion layer. This can be done by generating a model that contains the relevant layer dimensions and cloud radii as parameters. Fitting a large number of observed events to the model establishes the dimensions. Below we present a novel variation on this approach.

We now turn to measurements involving the laser plasma source.

\section{PLASMA SOURCE WORK}

An $200 \mu \mathrm{m}$ water jet under 1 atm He gas provides the basis of our laser plasma source, described elsewhere. ${ }^{12}$ The arrangement has great promise for in-house subpicosecond transmission extended $\mathrm{x}$-ray absorption fine structure (EXAFS), given the microbolometric detection scheme we are developing for it. ${ }^{12,29}$ Here, unless otherwise stated, we describe its $\mathrm{X}$-ray bremmstrahlung emissions when operated under aspirator vacuum. Such a vacuum is naturally constrained to water's vapor pressure, obviating boiling of the slightly cooled, degassed water that is fed from a reservoir under $1 \mathrm{~atm} \mathrm{He}$. A prototype apparatus can operate stably for hours at a time, but to date has been somewhat less reliable than the original pumped water jet under 1 atm He. X-ray spectral measurements using the vacuum apparatus have necessarily been based entirely on the CCD, since its use does not require exceptional long term stability. Characterization of the CCD has clearly been of critical importance in this approach. Observable x-ray yields are significantly increased under vacuum, and short laser pulses are relatively effective at producing hard $\mathrm{x}$ rays, motivating improved constructions.

The Ge detector in single photon mode can provide a spectrum of the $10 \mathrm{~Hz}$ repetition rate source. Long term shot to shot source stability and a few hours per spectrum are used to avoid pileup, but also requires the more reliable water jet in He arrangement for this particular measurement. At intervals during measurement, the Ge detector can be replaced with the CCD, which needs at most a few laser shots to generate a useful spectrum. The 1 pixel CCD events are 


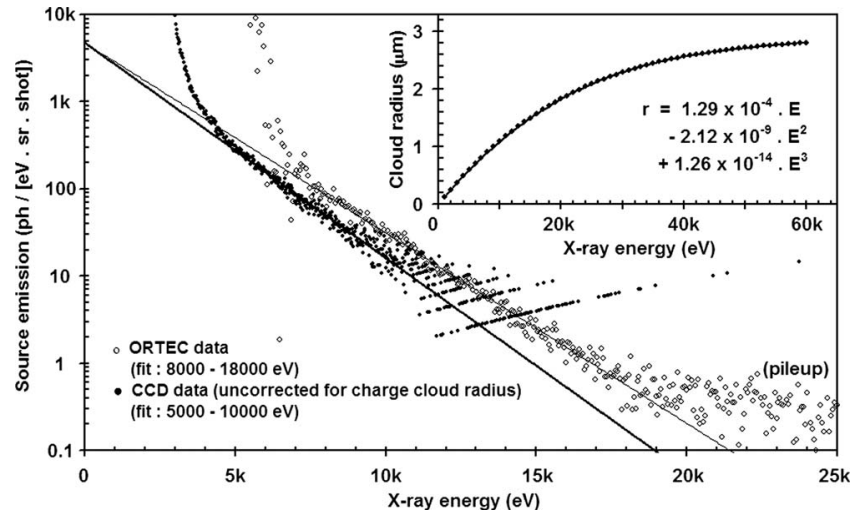

FIG. 3. Parallel measurement of an X-ray spectrum of the broadband laser plasma source using the He using the Ge point detector and the CCD, after normalizations for filters, pileup, energy binning interval, solid angle, and number of laser shots. Slope differences are due to larger electron clouds from high energy events being less able to fit in the CCD's depletion layer thickness. The CCD's depletion depth is adjusted to give a common zero energy extrapolation for both detectors, where the cloud size is expected to vanish. This interpretation allows quantitative estimation of the cloud size variation with energy, shown in the inset (see text).

isolated in software. Filters, pileup, energy binning intervals, solid angles, and number of laser shots are normalized for both detectors. The relatively insensitive surface layer of the CCD is included as an additional filter for the 1 pixel events, since these are associated with the thin depletion layer of the CCD. The earlier indication of 10.6 and $4.4 \mu \mathrm{m}$ obtained in the ${ }^{55} \mathrm{Fe}$ measurement provides a first guess of these thicknesses. Figure 3 presents parallel Ge and CCD spectra obtained in this way. Here, respective thicknesses of 9.2 and $5.8 \mu \mathrm{m}$ are applied to the CCD model, constraining the combined thickness to $15 \mu \mathrm{m}$. This choice is based on extrapolation of both spectra to a common point at the limit of low energy, where cloud radii are expected to vanish. We note that incorrect filter normalization leads to apparent nonlinearities at low energies (when shown on the log scale). Variation of the CCD depletion layer thickness in the range of 2-10 $\mu \mathrm{m}$ does not cause this effect to an objectionable extent (filters other than the CCD's surface layer dominate the low energy attenuation), but instead mostly determines the height of the CCD data on the plot, as it should.

Electron cloud radii and their variation with energy can be estimated from Fig. 3, as we now show.

The different detector slopes of the two detectors in Fig. 3 can be attributed to the fact that higher energy photons cause larger electron clouds, which are less likely to fall completely within our CCD's narrow depletion layer. To bring CCD spectra into agreement with corresponding $\mathrm{Ge}$ spectra requires increasing the number of observed 1 pixel events by an energy dependent factor $\exp (d E)$, where $d$ $\left(\sim 0.066 \mathrm{keV}^{-1}\right)$ is the difference of the two fitted gradients in the log plots. This factor, which we apply in all subsequent spectra, is expected to equal $p^{2} h /\left[(p-2 r)^{2}(h-2 r)\right]$ (model in Fig. 2), being the ratio of volumes within a pixel that describe the 1 pixel full registry losses when considering a cloud of finite size. Equality gives an analytical expression for electron cloud radius versus energy. The expression is cumbersome, but its validity is limited by data quality and
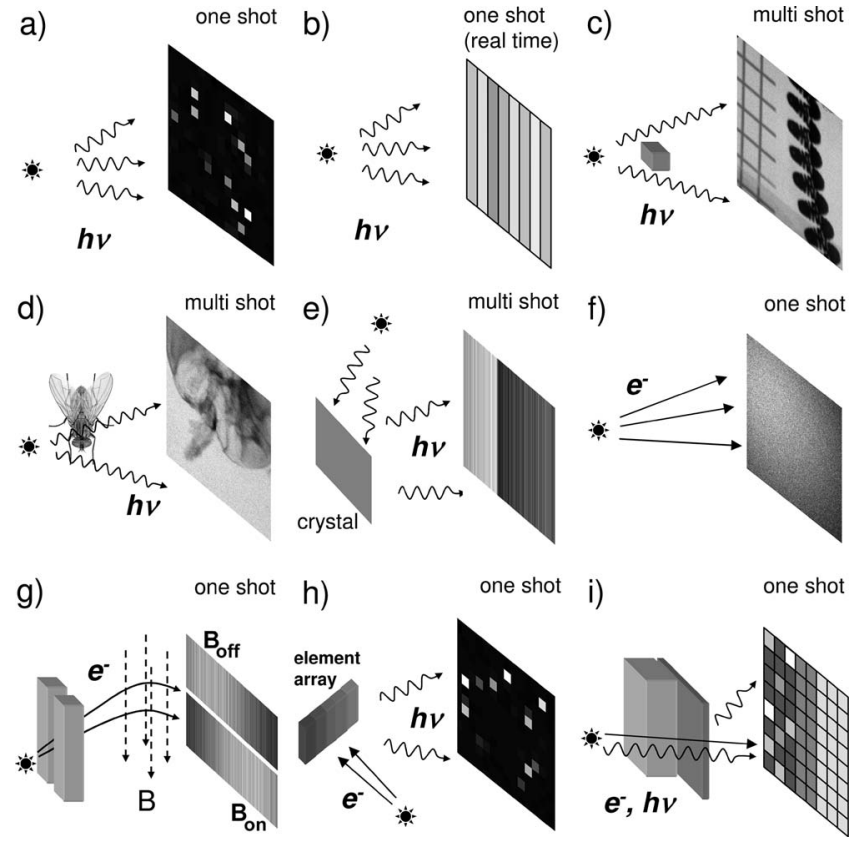

FIG. 4. Selection of laser plasma source experiments motivating CCD characterization. Requirement for single or multiple shots is indicated in the panels. Data measured using the CCD are shown in each case, except as follows; (a) X-ray photons impinge on the CCD in discrete pixels enabling histogram spectra. (b) Aggressive hardware binning allows real time optimization of x-ray flux (impression only, vertical binning sketched). (c) Blurred shadow edges of high contrast objects reveal source dimensions. (d) Biological specimens can be imaged by x-ray absorption. (e) Crystal dispersion of x-ray energies (von Hamos, absorption edge of Ti foil is illustrated, vertically binned). (f) Energetic electron beams are produced when using double or impaired temporal contrast laser pulses, traversing filters (with scatter and secondary radiation) to impinge directly on the CCD. (g) Magnetic deflection confirms electron beam polarity and energy. (h) Indirect observation of electron beams by x-ray fluorescence from element arrays. (i) Single shot distinction of $\mathrm{x}$ rays vs electron beams (CCD half obscured by thick absorber, other half by thin element foil; $x$ rays produce sharp edge shadow with absorption edge in foil transmission, electrons give a diffuse shadow (scatter) with $\mathrm{x}$-ray emission lines from the foil in the shadow; impression in figure applies to electron beam).

assumptions. We therefore provide a simple polynomial fit to the analytical electron cloud radius over an extended energy range where it might be of interest to do so, and urge a suitable level of awareness when using the result (inset of Fig. 3). The size of the cloud radii outside the fitted regions cannot be presumed with confidence. However, we note that extrapolation predicts $\sim 2.8 \mu \mathrm{m}$ cloud radius for the 59.5 $\mathrm{keV}$ photons provided by ${ }^{241} \mathrm{Am}$. Such events could be fully contained within the proposed $\sim 5.8 \mu \mathrm{m}$ potential well depth of the CCD and so be fully registered, though this would happen only rarely. Qualitatively, this is precisely what we observe.

At this point, we are in a strong position to apply the CCD to new and unknown x-ray sources, which can show diverse and exciting behavior. ${ }^{30-36}$ As Fig. 4 illustrates, laser plasma sources ${ }^{12}$ can fit this description very well, where we show a selection of our data obtained using this CCD. The characterization in this work assists in many ways. When working with $\mathrm{x}$-ray spectra, we have the necessary knowledge of the CCD's stopping power, spectral resolution, and limitations. Primary and secondary x-ray radiations are instantly recognizable, with clear identification of responsible 


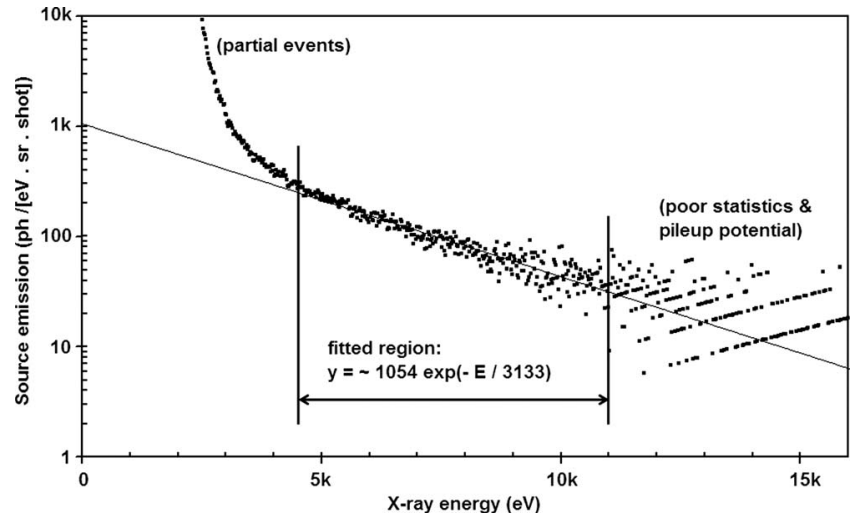

FIG. 5. Example of an x-ray spectrum from the laser plasma source when operated under aspirator vacuum, here using 38 laser shots at $4.0 \mathrm{~mJ} / \mathrm{pulse}$ with estimated pulse duration $\sim 118$ fs (see text).

elements in both emission and absorption measurements. In quantitative spectral flux work, we know to isolate 1 pixel events to minimize spectral redistribution, and have determined the functional depth structure associated with these (and multiple pixels) events. There is an appreciation of the effects of charge cloud size and its variation with energy. Observed spectra can be suitably compensated, enabling correct deduction of the source spectra. Noise levels can be anticipated in different operation modes. The extent of edge blur associated with the detector is known. If dealing with $\mathrm{x}$-ray images, we know that high levels of partial registry must be borne in mind when addressing intensities. Partially registered events that may be dispersed as a spectrum over the CCD area can be confidently interpreted. There can be no mistake when new radiations enter the picture, for example, energetic electron beams. In our work, their initial observation $^{12}$ was made using this CCD, and their study in a forthcoming publication has been significantly assisted by it, as illustrated. The CCD depth structure determined in this work is a prerequisite for calculations of the observable signals generated by the new electron-Si interactions.

We close with some recent measurements of laser plasma X-ray spectra generated under aspirator vacuum. The following measurements are valuable in applications presently under development and are entirely reliant on this CCD characterization.

Earlier laser plasma source work under He established that polarization, interaction geometry, and temporal contrast play critical roles for the bremsstrahlung x-ray (and electron beam) generation. ${ }^{12}$ To date the double laser pulse structrue ${ }^{37}$ that we have used ${ }^{12}$ to generate electron beams has not led to dramatic increases in primary $\mathrm{x}$-ray generation from our water jet target. Investigation of this matter has required careful distinction of the two radition types and their secondary radiations, which is enabled by the CCD as shown in Fig. 4. The same observations apply under vacuum. Figure 5 is representative of $\mathrm{x}$-ray generation from a water jet under vacuum, applying all the CCD corrections discussed above to a $4 \mathrm{~mJ} /$ pulse, $\sim 118$ fs duration, $780 \mathrm{~nm}$ Ti:sapphire generated x-ray spectrum, here compiled from 38 laser shots. Excluding the known detector limitations at either end of the fitted region, such fits are invariably excellent fits to a simple
TABLE II. Fits to $N=N_{0} \exp (-E / T)$ (photons/ per eV bandwidth per sr per laser shot) under aspirator vacuum, with fixed laser pulse duration (estimated $\sim 118 \mathrm{fs})$. The apparent anomaly at $6.0 \mathrm{~mJ}$ is noted in the text.

\begin{tabular}{cccc}
\hline \hline $\begin{array}{l}\text { Laser energy } \\
\text { per pulse }(\mathrm{mJ})\end{array}$ & $N_{0}(\mathrm{ph} / \mathrm{eV}$ sr shot $)$ & $T(\mathrm{eV})$ & Fit range $(\mathrm{eV})$ \\
\hline 1.0 & 420 & 1840 & $4500-8500$ \\
2.0 & 560 & 2300 & $4500-9000$ \\
3.0 & 850 & 2710 & $4500-10500$ \\
4.0 & 1050 & 3130 & $4500-11000$ \\
6.0 & 840 & 1800 & $4500-9000$ \\
10.0 & 1324 & 3290 & $5000-12000$ \\
20.0 & 1560 & 2750 & $5000-12000$ \\
\hline
\end{tabular}

exponential: $N=N_{0} \exp (-E / T)$. Compared to operation under $\mathrm{He}$, vacuum operation of the water jet gives significant increases in observable $\sim 4-20 \mathrm{keV}$ x-ray yields, as observed previously. ${ }^{12}$ The temperature parameter $(T)$ plays the dominant role in this increase. The enhancement is particularly useful at low laser pulse energies (such as when adapting to kilohertz repetition rate lasers), and when using near transform limited pulse durations.

The fit parameters listed in Tables II and III should guide the establishment of new x-ray sources of this kind. Laser pulse duration estimates are based on occasional calibrations of the optical compressor using an optical autocorrelator.

In Table II, fitted prefactors $\left(N_{0}\right)$ and temperatures $(T)$ are given for a range of laser pulse energies, at a constant laser pulse duration of $\sim 118$ fs. Such laser pulse energies are typically encountered in conventional optical laboratories using amplified $780 \mathrm{~nm}$ Ti:sapphire lasers. An interesting trend is that the production of $\mathrm{x}$ rays may begin to plateau above $\sim 4 \mathrm{~mJ} /$ pulse. This is welcome news when setting up on a kilohertz laser system, where the energy per pulse may otherwise be restrictive.

The duration of the laser pulse is another critical metric, partly because it affects the hard x-ray yield, and partly because it potentially determines the duration of the x-ray pulse. $^{12}$ In Table III, prefactors $\left(N_{0}\right)$ and temperatures $(T)$ are given for several laser pulse durations, at a constant laser energy of $4 \mathrm{~mJ} /$ pulse. Here an interesting observation is that the shortest available laser pulses give only slightly reduced yields of observable hard $\mathrm{x}$ rays. This contrasts with the situation under $\mathrm{He}$, where the same action leads to relatively dramatic reduction in observable $x$-ray yields. ${ }^{12}$ The presence of He gas evidently plays a role that goes beyond selffocusing, since the position of the jet in the laser focus was

TABLE III. Fits to $N=N_{0} \exp (-E / T)$ (photons/eV bandwidth per sr per laser shot) under aspirator vacuum, with fixed laser energy per pulse $(4.0 \mathrm{~mJ})$

\begin{tabular}{cccc}
\hline \hline $\begin{array}{c}\text { Pulse duration } \\
\text { (fs; estimate })\end{array}$ & $N_{0}(\mathrm{ph} / \mathrm{eV}$ sr shot $)$ & $T(\mathrm{eV})$ & Fit range $(\mathrm{eV})$ \\
\hline 38 & 640 & 3590 & $4500-10000$ \\
43 & 840 & 3330 & $4500-10000$ \\
54 & 1110 & 3550 & $4500-10000$ \\
65 & 1030 & 3340 & $4500-10000$ \\
118 & 1270 & 3090 & $4500-10000$ \\
\hline \hline
\end{tabular}


optimized with regard to observable $\mathrm{x}$-ray yield in every spectral measurement. At the same time, the observation indicates that measurable electron stopping ranges in the target are likely to determine the $\mathrm{x}$-ray pulse duration. ${ }^{12}$

Clearly, one seeks to map out the x-ray spectra as functions of many laser and experiment variables. Reproducibility is essential, and often obtained. For example, independent $4 \mathrm{~mJ}, \sim 118$ fs measurements appear in Tables II and III. They were made an hour apart, and are in reasonable agreement. However, practical difficulties delay such efforts and account for anomalies in the tabulated data. Thus, the $6 \mathrm{~mJ}$ $N_{0}$ and $T$ parameters shown in Table II would not be interpolated from the corresponding 4 and $10 \mathrm{~mJ}$ values; indeed some unexplained but temporary difficulties were noted with that measurement. Variability in experiments to date is believed to be dominated by the following causes. In our prototype vacuum apparatus, stability and reproducibility of the jet cannot be taken for granted, although the tiny stream appeared smooth and laminar on nearly all occasions when it was visually inspected. Proper degassing, slight cooling of incoming water, and leak-free plumbing are essential. Occasional small air leaks into the vacuum chamber or partial jet blockages might in principle lead to instability of the jet position and/or affect the laser focusing. Several degrees of freedom are needed in the critical manual alignment of the off-axis parabola. Once optimized (by generating a visible spark in air at the lowest possible energy at the start of each campaign), it is normally left alone, subsequently translating the target into the focal point; variations can be expected from one campaign to the next. Temporal aspects of the laser operation are known to critically affect laser-matter interactions and have led to constant maintenance and evolution of the laser system since its inception. ${ }^{17}$ Temperamental behavior of the laser system's many active components often do correlate with anomalous experiments in ways that can be more or less subtle, erratic or intermittent, and are not always easy to identify or remedy. Experimental variability clearly is a problem that can be improved in future work, meanwhile a degree of caution needs to be exercised when inferring trends.

\section{CONCLUSION}

Characterization of a backthinned, backilluminated CCD has enabled quantitative investigation of $\sim 4-20 \mathrm{keV}$ x-ray emissions from a laser plasma radiation source. Its responses to monochromatic and multichromatic radioactive $\mathrm{x}$-ray sources, as well as pulsed isotropic broadband $\mathrm{x}$ rays from the laser plasma source have been optimized, interpreted, and quantified for practical use. Collectively, results lead to appreciation of a partially sensitive surface layer of $9.2 \mu \mathrm{m}$, beneath which is a quantitatively sensitive depletion layer of $5.8 \mu \mathrm{m}$. This is consistent with limited available manufacturer information and other descriptions of CCDs where the internal structure was known. The particular CCD topology is uncommon within the x-ray single photon community, since the relatively thick and exposed surface layer scrambles events to lower energies, while the depletion layer is rather thin and deeply buried. Nevertheless we success- fully extract quantitative hard $\mathrm{x}$-ray spectra by directing attention to events isolated in single pixels. Further, we infer the variation of nascent electron cloud radii in Si by x-ray photons spanning a continuum of energies, by observations of broadband spectra from the laser plasma source. The approach takes advantage of the thinness of the depletion layer. We illustrate the CCDs use in a range of laser plasma X-ray experiments, where we indicate the significance of the present characterization. Finally, we show valuable new $\mathrm{x}$-ray spectral flux measurements that are entirely reliant on the CCD and its characterization. These measurements are of immediate value when setting up similar laser plasma sources using ultrafast lasers capable of a few millijoules per pulse. Such developments, in conjunction with microbolometer detectors, are expected to enable laboratory based ultrafast EXAFS on a very widespread scale. ${ }^{12}$

\section{ACKNOWLEDGMENTS}

We wish to thank C.-G. Wahlström and Lund Tekniska Högskolans, Department of Atomic Physics for access to the Terawatt Laser Facility and the CCD used in this work. We especially wish to thank the following individuals, who have been particularly helpful in assisting with day-to-day laser operation over a period of several years: A. Persson, E. Pourtal, O. Lundh, F. Lindau, Y. Glinec, G. Genoud, and M. Harbst. This work has benefited through the Swedish Consortium for Artificial Photosynthesis, Sweden's Kungliga Fysiografiska Sällskap, the Swedish Energy Agency (STEM), the Knut and Alice Wallenberg Foundation, and a Linneus grant awarded to Lund's Laser Centre. J. Uhlig and M. Walczak acknowledge financing through the MAXlas project, under the Sixth European Framework Programme (contract number MEST-CT-2005-020356).

${ }^{1}$ J. R. Janesick, Scientific Charge-Coupled Devices (SPIE, Bellingham, WA, 2001).

${ }^{2}$ L. Labate, A. Giulietti, D. Giulietti, P. Koster, T. Levato, L. A. Gizzi, F. Zamponi, A. Lubcke, T. Kampfer, I. Uschmann, and E. Forster, Rev. Sci. Instrum. 78, 103506 (2007)

${ }^{3}$ B. E. Burke, J. A. Gregory, M. W. Bautz, G. Y. Prigozhin, S. E. Kissel, B. B. Kosicki, A. H. Loomis, and D. J. Young, IEEE Trans. Electron Devices 44, 1633 (1997)

${ }^{4}$ H. Tsunemi, E. Miyata, H. Ozawa, D. Matsuura, H. Tomida, H. Katayama, and K. Miyaguchi, Nucl. Instrum. Methods Phys. Res. A 579, 866 (2007).

${ }^{5}$ C. M. Laperle, P. Wintermeyer, J. R. Wands, D. Shi, M. A. Anastasio, X. Li, B. Ahr, G. J. Diebold, and C. Rose-Petruck, Appl. Phys. Lett. 91, 173901 (2007).

${ }^{6}$ S. C. Mayo, P. R. Miller, S. W. Wilkins, T. J. Davis, D. Gao, T. E. Gureyev, D. Paganin, D. J. Parry, A. Pogany, and A. W. Stevenson, J. Microsc. 207, 79 (2002).

${ }^{7}$ C. Broennimann, E. F. Eikenberry, B. Henrich, R. Horisberger, G. Huelsen, E. Pohl, B. Schmitt, C. Schulze-Briese, M. Suzuki, T. Tomizaki, H. Toyokawa, and A. Wagner, J. Synchrotron Radiat. 13, 120 (2006).

${ }^{8}$ A. Rousse, P. Audebert, J. P. Geindre, F. Fallies, J. C. Gauthier, A. Mysyrowicz, G. Grillon, and A. Antonetti, Phys. Rev. E 50, 2200 (1994).

${ }^{9}$ F. Zamponi, T. Kampfer, A. Morak, I. Uschmann, and E. Forster, Rev. Sci. Instrum. 76, 116101 (2005).

${ }^{10}$ T. Lee, Y. Jiang, C. G. Rose-Petruck, and F. Benesch, J. Chem. Phys. 122, 084506 (2005)

${ }^{11}$ J. Chen, H. Zhang, I. V. Tomov, M. Wolfsberg, X. Ding, and P. M. Rentzepis, J. Phys. Chem. A 111, 9326 (2007).

${ }^{12}$ W. K. Fullagar, M. Harbst, S. Canton, J. Uhlig, M. Walczak, C. G. Wahlstrom, and V. Sundstrom, Rev. Sci. Instrum. 78, 115105 (2007).

${ }^{13}$ C. Tillman, PhD Thesis, Lunds Universitet, 1996, LRAP-204.

${ }^{14}$ G. Grätz, PhD Thesis, Lunds Universitet, 1998, LRAP-236. 
15 A. Sjögren, PhD Thesis, Lunds Universitet, 2002, LRAP-288.

${ }^{16}$ E. Danielsson, Masters Thesis, Lunds Universitet, 2003, LRAP-309.

${ }^{17}$ S. Svanberg, J. Larsson, A. Persson, and C.-G. Wahlström, Phys. Scr. 49, 187 (1994).

${ }^{18}$ XCOM, Photon Cross Sections Database, http://physics.nist.gov/ PhysRefData/Xcom/Text/XCOM.html

${ }^{19}$ M. J. Berger, J. S. Coursey, M. A. Zucker, and J. Chang, ESTAR: Electron Stopping-Power and Ranges, http://physics.nist.gov/PhysRefData/Star/ Text/ESTAR.html

${ }^{20}$ U. Fano, Phys. Rev. 72, 26 (1947).

${ }^{21}$ Ch. Enss, Cryogenic Particle Detection, Topics in Applied Physics (Springer, New York, 2005), Vol. 99.

${ }^{22}$ G. C. Holst and T. S. Lomheim, CMOS/CCD Sensors and Camera Systems (JCD, Winter Park, FL/SPIE, Bellingham, WA, 2007).

${ }^{23}$ S. E. Holland, D. E. Groom, N. P. Palaio, R. J. Stover, and M. Wei, IEEE Trans. Electron Devices 50, 225 (2003).

${ }^{24}$ G. Prigozhin, N. R. Butler, S. E. Kissel, and G. R. Ricker, IEEE Trans. Electron Devices 50, 246 (2003).

${ }^{25}$ A. Shariff, B. G. Martinsson, V. Auzelyte, M. Elfman, P. Kristiansson, K. G. Malmqvist, C. Nilsson, J. Pallon, and M. Wegden, Nucl. Instrum. Methods Phys. Res. B 219, 110 (2004).

${ }^{26}$ W. Kester, Which ADC Architecture is Right for Your Application? http:// www.analog.com/library/analogdialogue/archives/39-06/architecture.html

${ }^{27}$ M. W. Bautz, G. Y. Prigozhin, M. J. Pivovaroff, S. E. Jones, S. E. Kissel, and G. R. Ricker, Nucl. Instrum. Methods Phys. Res. A 436, 40 (1999).

${ }^{28}$ G. Prigozhin, S. Jones, M. Bautz, G. Ricker, and S. Kraft, Nucl. Instrum. Methods Phys. Res. A 439, 582 (2000).

${ }^{29}$ I. J. Maasilta, K. M. Kinnunen, A. K. Nuottajärvi, J. Leppäniemi, and A. Luukanen, Supercond. Sci. Technol. 19, S242 (2006).

${ }^{30}$ A. Rousse, K. T. Phuoc, R. Shah, A. Pukhov, E. Lefebvre, V. Malka, S. Kiselev, F. Burgy, J.-P. Rousseau, D. Umstadter, and D. Hulin, Phys. Rev. Lett. 93, 135005 (2004).

${ }^{31}$ E. Sato, E. Tanaka, H. Mori, T. Kawai, T. Ichimaru, S. Sato, K. Takayama, and H. Ido, Med. Phys. 32, 49 (2005).

${ }^{32}$ C. N. Boyer, G. E. Holland, and J. F. Seely, Rev. Sci. Instrum. 76, 035109 (2005).

${ }^{33}$ L. Soto, Plasma Phys. Controlled Fusion 47, A361 (2005).

${ }^{34}$ E. J. Lerner and A. Blake, U.S. Patent No. 20070201598A1 (2007).

${ }^{35}$ H. Daido, Rep. Prog. Phys. 65, 1513 (2002).

${ }^{36}$ J. Seres, P. Wobrauschek, Ch. Streli, V. S. Yakovlev, E. Seres, F. Krausz, and Ch. Spielmann, New J. Phys. 8, 251 (2006).

${ }^{37}$ K. Hatanaka, H.. Ono, and H. Fukumura, Appl. Phys. Lett. 93, 064103 (2008). 